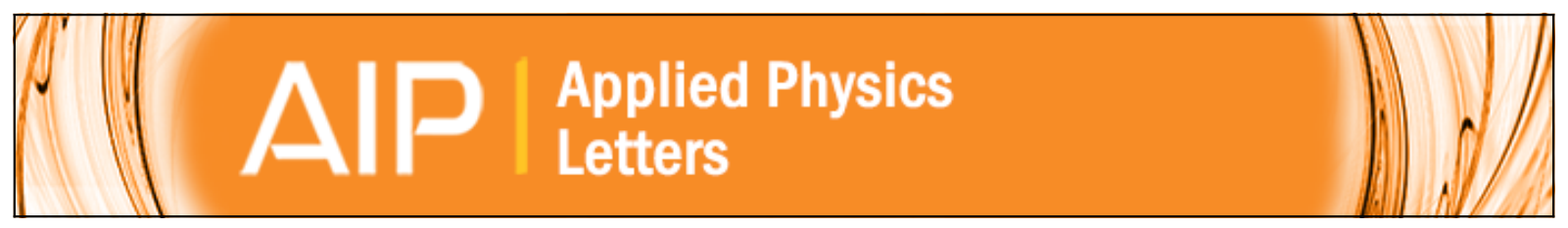

Formation of shallow acceptor states in the surface region of thin film diamond

Oliver A. Williams, Michael D. Whitfield, Richard B. Jackman, John S. Foord, James E. Butler, and Christoph E. Nebel

Citation: Applied Physics Letters 78, 3460 (2001); doi: 10.1063/1.1345806

View online: http://dx.doi.org/10.1063/1.1345806

View Table of Contents: http://scitation.aip.org/content/aip/journal/apl/78/22?ver=pdfcov

Published by the AIP Publishing 


\title{
Formation of shallow acceptor states in the surface region of thin film diamond
}

\author{
Oliver A. Williams, Michael D. Whitfield, and Richard B. Jackman ${ }^{\text {a) }}$ \\ Electronic and Electrical Engineering, University College London, Torrington Place, London, \\ WC1E 7JE United Kingdom \\ John S. Foord \\ Physical and Theoretical Chemistry, University of Oxford, South Parks Road, Oxford, \\ OX1 3QZ United Kingdom \\ James E. Butler \\ Code 6174, Naval Research Laboratory, Washington, DC 20375 \\ Christoph E. Nebel \\ Walter Schottky Institut, TU-Muenchen, AM Coulombwall, D-80469 Muenchen, Germany
}

(Received 26 July 2000; accepted for publication 29 November 2000)

\begin{abstract}
Considerable interest exists in fabrication of electronic devices from thin film polycrystalline diamond. To date, doping this material to achieve good free carrier concentrations and mobilities at room temperature has proved difficult. In this letter we report low temperature Hall effect measurements made on diamond films subjected to a hydrogenation process, such that the near surface region becomes $p$ type without the addition of conventional dopant atoms. High carrier concentrations and mobilities can be achieved. The change in carrier concentration within the temperature range $10-300 \mathrm{~K}$ does not change as expected for most films, actually increasing as the temperature falls. This effect could be related to the confinement of carriers at the surface caused by the dipole provoked by adsorbed hydrogen on the diamond. However, polished films display more conventional behavior in that the carrier concentration falls with falling temperature. (C) 2001 American Institute of Physics. [DOI: 10.1063/1.1345806]
\end{abstract}

The emergence of chemical vapor deposition (CVD) methods for the formation of large area polycrystalline diamond films ${ }^{1}$ has led to considerable interest in the use of this material within electronic applications. For most electronic devices doped material is required. Whilst boron $^{1}$ and phosphorus $^{2}$ act as acceptor and donor states, respectively, these species form deep levels $(0.37$ and $0.43 \mathrm{eV})$ and, hence, yield few free carriers at room temperature. It has recently been demonstrated that near surface hydrogen promotes the formation of acceptor states in this region of CVD diamond films. ${ }^{3-5}$ We have previously studied the room temperature characteristics of these acceptor states, in terms of carrier concentrations and mobility values derived from Hall measurements. ${ }^{6}$ The highest room temperature hole mobility within polycrystalline diamond reported to date was measured (at, for example, $70 \mathrm{~cm}^{2} \mathrm{~V}^{-1} \mathrm{~s}^{-1}$ for a carrier concentration of $5 \times 10^{17} \mathrm{~cm}^{-3}$ ). Whilst the origin of these carriers remains controversial, our recent studies suggest that the interaction of hydrogen with defects within the near surface region of CVD material leads to the formation of very shallow acceptor states. ${ }^{7}$ This approach to doping thin film polycrystalline diamond has been successfully used to produce highly effective field effect transistors. ${ }^{8}$ In this letter we report low temperature Hall effect measurements performed on a number of differing types of CVD diamond, each of which

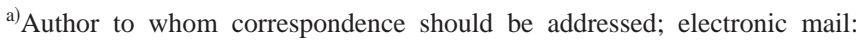
r.jackman@ee.ucl.ac.uk
}

has been subjected to a hydrogenation process. The results achieved yield important insight into the nature of the acceptor states that are formed by hydrogenation.

Free standing diamond films, grown by microwave plasma enhanced CVD, were used throughout this study. During Raman spectroscopy all films revealed an intense peak at $1332 \mathrm{~cm}^{-1}$, indicative of good quality diamond, ${ }^{1}$ with no other structure being evident. All films displayed prominent crystal faceting, with random grain alignments, but differed in grain size. The characteristics of these samples (A, B, and C) in terms of thickness and grain size are given in Table I. Sample C1 and C2 were identical, but sample type $\mathrm{C} 2$ had been mechanically polished using diamond grit bonded in a resin matrix. Subsequent acid and $\mathrm{H}$ plasma treatments are believed to remove superficial damage and nondiamond carbon, leaving the surface covered with submicroscopic (111) and (100) facets, giving rise to an optically smooth surface. ${ }^{9,10}$ All films were initially immersed in a strongly oxidizing solution which is known to leave a contaminant free surface. ${ }^{11}$ A pure hydrogen microwave plasma $(2.45 \mathrm{GHz}, 800 \mathrm{~W}, 40$ Torr, $5 \mathrm{~min}$, sample tempera-

TABLE I. The characteristics of the sample types used in this study.

\begin{tabular}{cccc}
\hline \hline Sample type & Thickness $(\mu \mathrm{m})$ & Grain size $(\mu \mathrm{m})$ & Notes \\
\hline A & 300 & $40-60$ & \\
B & 100 & $10-30$ & \\
C1 & 100 & $20-40$ & \\
C2 & 100 & $20-40$ & As C1, but polished \\
\hline \hline
\end{tabular}




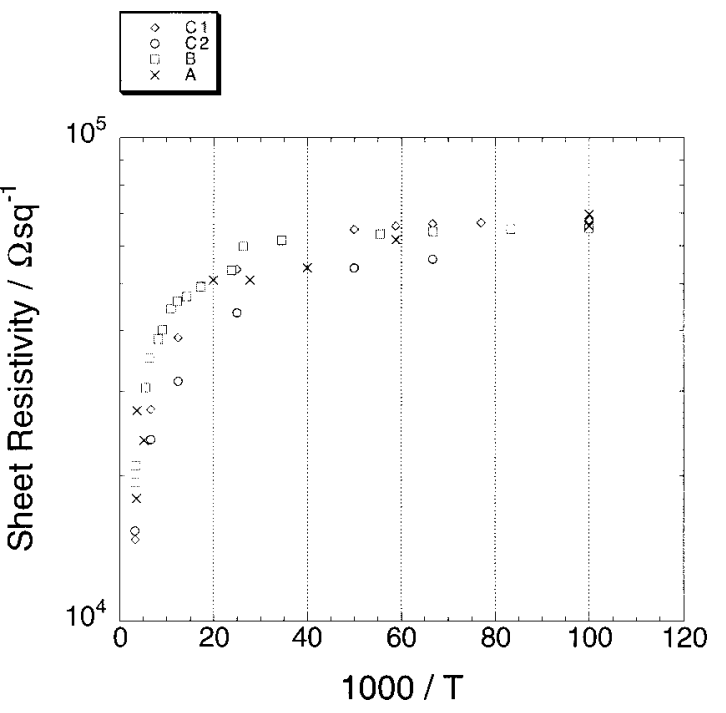

FIG. 1. Sheet resistivity of the samples studied plotted as a function of sample temperature.

ture $500{ }^{\circ} \mathrm{C}$ ) was then used to hydrogenate each of the samples. Hall effect measurements were carried out using the four point probe Van Der Pauw method for conformal mapping at temperatures between 10 and $300 \mathrm{~K}$, using a magnetic field strength of $2 \mathrm{~T}$. Evaporated gold contacts were used throughout, which displayed ohmic $I-V$ characteristics on these films.

All of the samples tested were $p$ type, as determined from their positive Hall coefficients. The measurements performed yield the sheet resistivity and sheet carrier concentration, from which carrier mobility values can be determined. The values obtained for each sample type can be seen in Figs. 1-3, plotted as a function of temperature. The sheet resistivity of all samples (Fig. 1) increases with decreasing temperature. Whilst the values for all sample types vary between $10^{4}$ and $10^{5} \Omega \mathrm{cm}^{-2}$, the values for $\mathrm{C} 2$ are consistently lower than those for the others, indicating that this material remains more conductive over this temperature range. There is even more diversity in the carrier concentrations measured (Fig. 2). Sheet carrier concentrations for C1

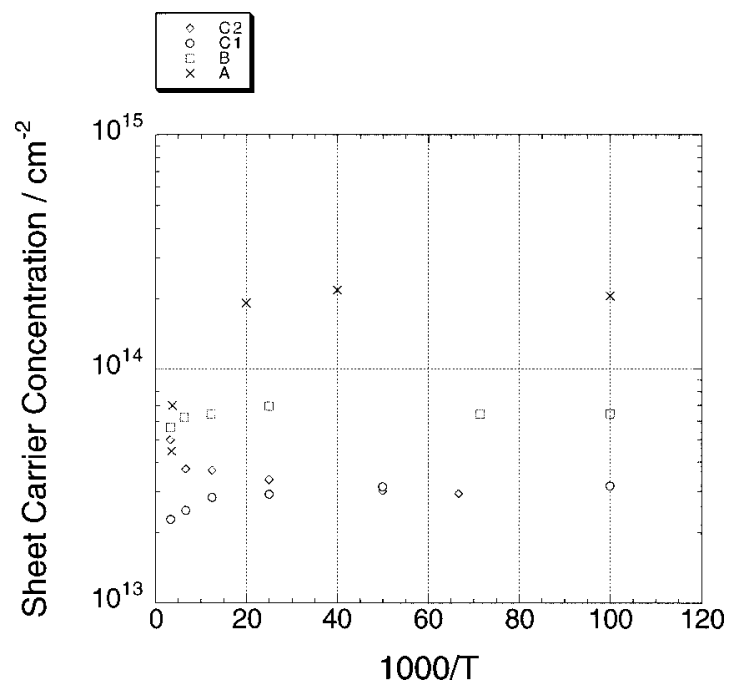

FIG. 2. Sheet carrier concentration for the samples studied plotted as a function of sample temperature.

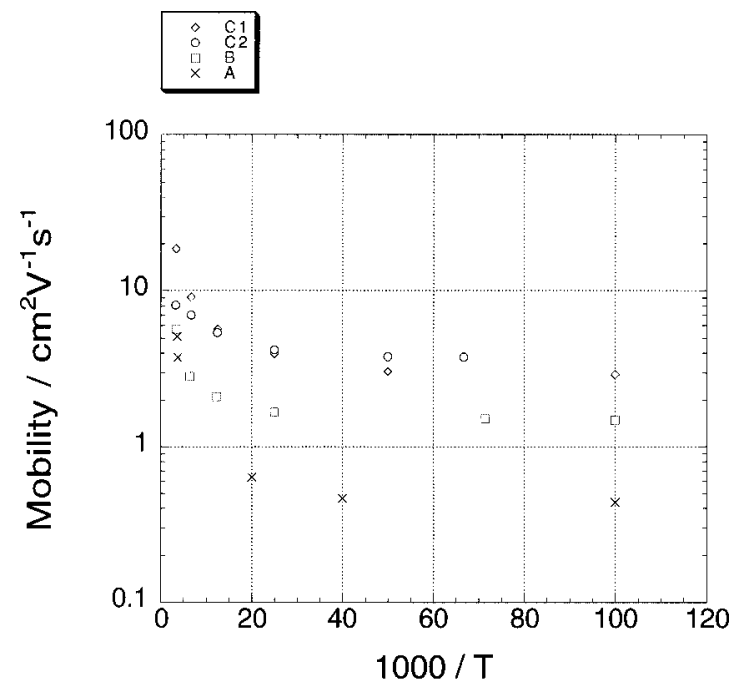

FIG. 3. Carrier mobility values for the samples studied plotted as a function of sample temperature.

and $\mathrm{C} 2$ (at around $3 \times 10^{13} \mathrm{~cm}^{-2}$ ) can be seen to be around a factor of three lower than B, and up to a factor of ten lower than A. The transistor characteristics for devices formed on this type of material suggest that the carriers are dispersed throughout a layer of up to $20 \mathrm{~nm}$ in depth. ${ }^{12}$ This implies carrier concentrations as high as $10^{19} \mathrm{~cm}^{-3}$ are being achieved in these samples. The variation in carrier concentration with temperature differs markedly in the case of $\mathrm{C} 2$, compared to all of the other sample types. $\mathrm{C} 2$ shows a decrease in carrier concentration as temperature decreases whilst the other sample types actually show a slight increase with decreasing temperature, over the range studied here. The carrier mobility values determined for all samples, within the temperature range 10-300 K, are shown in Fig. 3 . This plot again reveals significant differences in the values recorded for each of the types of CVD diamond films. All mobility values decrease with decreasing temperature, with values between 0.5 and $20 \mathrm{~cm}^{2} \mathrm{~V}^{-1} \mathrm{~s}^{-1}$. At $300 \mathrm{~K}, \mathrm{C} 1 \mathrm{re}-$ veals the highest mobility value, followed by $\mathrm{C} 2, \mathrm{~B}$, and $\mathrm{A}$. However, the carrier mobility recorded for $\mathrm{C} 2$ decreases less with decreasing temperature than does the same diamond type that has not been polished, assuming mobility values that are similar to the unpolished $\mathrm{C} 1$ at temperatures of 100 $\mathrm{K}$ and below.

These results are intriguing. According to conventional semiconductor theory, the value of the carrier concentration should remain constant with temperature, until the point at which the available energy is insufficient for activation, when it begins to decline. This is the case here only for $\mathrm{C} 2$. If the carriers in this sample are assumed to have a conventional origin, the data in Fig. 3 imply the activation energy for these acceptor states must be less than $2 \mathrm{meV}$, which is extremely shallow. In all of the other sample types the carrier concentration actually appears to increase with decreasing temperature; this was a clear and persistent trend in these sample types. A possible explanation for this effect is the influence of adsorbed, as opposed to near surface, hydrogen on the carrier distribution within the material. Hydrogen termination of a diamond surface is known to provoke the formation of a surface dipole, causing band bending in this 
region of the material. ${ }^{13,14}$ This will act to confine any carriers present to the surface of the material. This confinement will affect the measured Hall voltage and, hence, the apparent carrier concentration determined using this technique. Since this confinement will be strongest at low temperatures, an increase in carrier concentration may be observed as the temperature is decreased. However, since no carrier "freezeout" is observed down to $10 \mathrm{~K}$, this explanation for the observed trends would suggest a carrier activation energy of less than $2 \mathrm{meV}$.

Similar trends in carrier concentration variation with temperature have been reported in Hall measurements performed on $\mathrm{Ge}^{15}$ and $\mathrm{GaN},{ }^{16,17}$ where an alternative explanation was put forward. Mott and Twose ${ }^{15}$ studied both $n$ - and $p$-type $\mathrm{Ge}$, and proposed a model involving transport through both defect centers and the conduction band (for $n$-type material) to explain the phenomenon. The conduction in the defect centers was considered to be either diffusive, due to the small but finite overlap of the localized electron wave functions of the defect centers, or hopping. The defect band mobility is expected to be small compared to the conduction band mobility, implying defect band conduction becomes dominant when the carrier concentration in the conduction band becomes negligible, i.e., at low temperatures. In the case of $n$-type GaN, Molnar et al. ${ }^{16}$ also found that transport through the conduction band and the auto-doping defect centers, at around $20-30 \mathrm{meV}$ below the conduction band, were important in explaining the measured transport characteristics. They observed a peak in the Hall coefficient at around $100 \mathrm{~K}$.

In the present case carrier (hole) concentrations measured for $\mathrm{A}, \mathrm{B}$, and $\mathrm{C} 1$ diamond sample types show an increasing level of carrier concentration with decreasing temperature, throughout the whole temperature range studied here $(10-300 \mathrm{~K})$. If the model discussed before is used to explain this trend, then transport in the valance band must be assumed to be progressively declining, becoming dominated by impurity band conduction at these low temperatures. However, this is curious since the carrier mobility values determined remain high for a polycrystalline material, which is not expected for impurity band transport. In the case of $\mathrm{C} 2$, the carrier concentration is found to be declining as the temperature decreases to $100 \mathrm{~K}$. At temperatures below this, only a very slight reduction in carrier concentration is observed. This implies that between 300 and $100 \mathrm{~K}$, valance band conduction may be dominant, giving way to impurity band conduction at lower temperatures. The data presented in Fig. 2 is not detailed enough to calculate the activation energy of the acceptor state and the respective concentrations in the valance and impurity bands. However, the decline in carrier concentration from 300 to $100 \mathrm{~K}$, if due to freeze-out of the carriers in the valance band, would suggest an acceptor state activation energy in the range $10-40 \mathrm{meV}$. A detailed analysis of the carrier transport data is being carried out and will be reported elsewhere.

At lower temperatures carriers spend longer in the vicinity of any impurities that are present and, hence, are more heavily scattered. This explains the decrease in mobility values with decreasing temperature. Since an increase in the concentration of impurity (acceptor) states increases the carrier scattering, the mobility values should also decrease with increasing carrier concentration at a given temperature. It is clear that as A has the highest carrier concentration at $300 \mathrm{~K}$, the lowest mobility values would be expected at this temperature, as is the case (Fig. 3). The films with smaller grain sizes support lower carrier concentrations, and have correspondingly higher mobilities. $\mathrm{C} 2$ has a different room temperature mobility value to the similar, but unpolished, $\mathrm{C} 1$, but becomes similar to $\mathrm{C} 1$ at temperatures of $100 \mathrm{~K}$ and below. This appears to support the assertion that $\mathrm{C} 2$ has two differing dominant conduction paths, possibly valance and impurity band, over this temperature range, whilst the other sample types may be dominated by only one.

The damage that is inevitably imparted to a diamond substrate when it is polished does not eradicate the hydrogen-based creation of $p$-type character, but rather makes it behave differently. There is clearly a need for further study into the carrier transport characteristics displayed by hydrogenated diamond films following a systematic variation in surface treatment conditions.

The authors are grateful to the Engineering and Physical Sciences Research Council (EPSRC) within the UK for financial support. One of them (O.A.W.) gratefully acknowledges M. Beck and UCL for the award of a Beck Scholarship. Alison M. Mainwood, Gordon Davies, and Carl Johnston at KCL are also acknowledged for useful discussions throughout this study.

${ }^{1}$ G. Sh. Gildenblat, S. A. Grot, and A. R. Badzian, Proc. IEEE 79, 647 (1991).

${ }^{2}$ S. Koizumi, H. Ozaki, M. Kamo, Y. Sako, and T. Inuzuka, Appl. Phys. Lett. 71, 1065 (1997).

${ }^{3}$ H. Kawarada, M. Aoki, H. Sasaki, and K. Tsugawa, Diamond Relat. Mater. 57, 718 (1994).

${ }^{4}$ H. Kawarada and M. Itoh, Jpn. J. Appl. Phys., Part 1 34, 4677 (1995).

${ }^{5}$ B. L. Mackey, J. N. Russell, Jr., J. E. Crowell, and J. E. Butler, Phys. Rev. B 52, R17009 (1995).

${ }^{6}$ H. J. Looi, J. S. Foord, and R. B. Jackman, Appl. Phys. Lett. 72, 353 (1998).

${ }^{7}$ H. J. Looi, L. Y. S. Pang, A. B. Molloy, F. Jones, J. S. Foord, and R. B. Jackman, Diamond Relat. Mater. 7, 550 (1998).

${ }^{8}$ H. J. Looi, L. Y. S. Pan, Y. Wang, M. D. Whifield, and R. B. Jackman, IEEE Electron Device Lett. 19, 112 (1998).

${ }^{9}$ B. D. Thoms, M. S. Owens, J. E. Butler, and C. Spiro, Appl. Phys. Lett. 65, 2957 (1994).

${ }^{10}$ T. W. Mercer, J. N. Russell, and P. E. Pehrsson, Surf. Sci. Lett. 392, L21 (1998).

${ }^{11}$ B. Baral, S. S. M. Chan, and R. B. Jackman, J. Vac. Sci. Technol. A 14, 1 (1996).

${ }^{12}$ H. J. Looi, L. Y. S. Pang, Y. Wang, M. D. Whitfield, and R. B. Jackman, Diamond Relat. Mater. 7, 565 (1998).

${ }^{13}$ J. Ristein, Diamond Relat. Mater. 9, 1129 (2000).

${ }^{14}$ A. Denisenko, A. Aleksov, A. Pribil, P. Gluche, W. Ebert, and E. Kohn, Diamond Relat. Mater. 9, 1138 (2000).

${ }^{15}$ N. F. Mott and T. D. Twose, Adv. Phys. 10, 107 (1961).

${ }^{16}$ R. J. Molnar, T. Lei, and T. D. Moustakas, Appl. Phys. Lett. 62, 72 (1993).

${ }^{17}$ D. C. Look and R. J. Molnar, Appl. Phys. Lett. 70, 3377 (1997). 